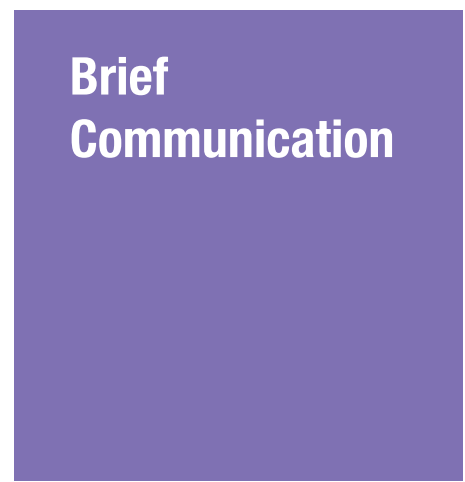

Submitted: 25 Apr 2016 Accepted: 8 Jun 2016 Online: 5 Oct 2016

\section{There are No Differences between Factors Determining Graft Infection in Autologous Bone Flap Replacement and Acrylic Cranioplasty: A Prospective Observational Study at Hospital Kuala Lumpur}

\author{
Siti Azleen Mohamad ${ }^{1,2,3}$, Mohd Safari Mohd Haspani ${ }^{1,2,3}$, \\ Badrisyah IDRIS ${ }^{2,3}$
}
$1 \quad$ Institut Kajisaraf Tunku Abdul Rahman (IKTAR), Hospital Kuala Lumpur, 50586 Jalan Pahang, Kuala Lumpur, Malaysia
2 Department of Neurosciences, School of Medical Sciences, Universiti Sains Malaysia, Jalan Hospital Universiti Sains Malaysia, 16150 Kubang Kerian, Kota Bharu, Kelantan, Malaysia
3 Department of Neurosciences, Hospital Universiti Sains Malaysia, Jalan Hospital Universiti Sains Malaysia, 16150 Kubang Kerian, Kota Bharu, Kelantan, Malaysia

To cite this article: Mohamad SA, Mohd Haspani MS, Idris B. There are no differences between factors determining graft infection in autologous bone flap replacement and acrylic cranioplasty: a prospective observational study at Hospital Kuala Lumpur. Malays J Med Sci. 2016;23(5): 83-90. http://dx.doi.org/10.21315/mjms2016.23.5.11

To link to this article: http://dx.doi.org/10.21315/mjms2016.23.5.11

\begin{abstract}
Objectives: This study, conducted in 2012, was performed to determine the incidence of graft infection after cranioplasty procedures and factors affecting the graft infection rate at Hospital Kuala Lumpur (HKL).

Methodology: This was an observational cross-sectional case study of patients who have undergone cranioplasty at HKL over a period of one year (2012). One hundred seventytwo patients were included in the study. A total of $105(61.8 \%)$ cases were autologous bone flap replacements, and $67(38.2 \%)$ were acrylic cranioplasty.

Result: A total of five infected grafts were identified among the 172 cases included in the study, resulting in an overall infection rate of $\mathbf{2 . 9 \%}$. Of this infected group, three (4.5\%) were cases of cranioplasty and two (1.9\%) were cases of autologous bone flap replacement. There was high proportion of male patients undergoing cranioplasty (118 [or 68.6\%]) and only 54 (or $31.4 \%)$ female patients. The primary pathology in the majority of patients (126 [or 73.2\%]) was due to trauma; only 46 cases (or 26.8\%) did not result from trauma. Of the patients, 123 (or 71.5\%) had undergone a single cranial procedure prior to their cranioplasty, and 43 (or $28.5 \%)$ had undergone multiple cranial procedures. Most of the patients (114 [or 66.3\%]) underwent cranioplasty 90 days after undergoing decompressive craniectomy while 58 (or $33.7 \%$ ) underwent cranioplasty less than 90 days after decompressive craniectomy.
\end{abstract}

Keywords: graft, infection, cranium, neuroscience

\section{Introduction}

Cranioplasty is defined as a surgical repair of acquired or congenital cranial defects. It is performed mainly for anatomical reconstruction, brain protection and cosmetic purposes (1).
Cranioplasty is commonly performed following craniectomy or decompressive craniectomy for traumatic brain injury. Performing a cranioplasty with the aim of reconstructing skull defects is, in essence, an art, and it poses certain challenges to neurosurgeons. The procedure requires thorough 
anatomical knowledge and meticulous surgical techniques and, when performed well, results in a good outcome in terms of cosmetics and anatomical protection. On the other hand, when performed incorrectly, it may lead to potentially serious complications, such as extradural or subdural collection, graft infection, graft failure, post-operative hematoma, edema and seizures (2).

Cranioplasty, like any surgical procedure, is associated with complications. Common complications include surgical site infection, graft infection, graft failure and poor cosmesis. Among these, graft infection has been most commonly documented. Over the years, cranioplasty has evolved in terms of both procedural techniques and materials to improve outcomes. Despite these improvements, the rate of graft infection remains significant. This has been attributed to many factors, including graft type, duration of surgery, the patient's premorbid condition, underlying disease and the sterility and environment of the operating room (3-6).

Surgical site infection, defined as infection resulting in surgical implantation of a bone flap, is a notorious complication that occurs in association with cranioplasty (6). This study aims to identify rates of infection after cranioplasty utilising both autologous cryopreserved bone grafts and methyacrylate grafts as well as factors associated with graft infection among cranioplasty patients.

\section{Patients and Methodology}

\section{Study design}

\section{Objectives}

The general objectives were to determine the incidence of wound infection after cranioplasty procedures in the neurosurgical department at Hospital Kuala Lumpur (HKL) as well as to observe the relationship between various risk factors affecting graft infection in cranioplasty.

The specific objectives were to identify risk factors of surgical site infection among patients who have undergone cranioplasty as well as to determine the association of factors such as implant type, gender, underlying primary pathology, number of prior cranial procedures, time interval to cranioplasty and pre-operative Glasgow Coma Score (GCS) with the development of surgical site infection among patients who have undergone cranioplasty.
This was an observational cross-sectional study on patients who have undergone cranioplasty at HKL over period of one year (2012). Cranioplasty is a surgical procedure involving the use of autologous bone or an acrylic flap to repair skull defects. The number of patients admitted to HKL for elective cranioplasty from January to December 2012 was obtained from the admissions office. The total number of patients admitted from the specified period was 172 . Some of these were referred from other hospitals and admitted to our neurosurgical clinic and ward.

HKL has been using a computer database system called Computerised Operating Theatre Documentation System (COTDS) in the operation theatre (OT) since 1999. With this Microsoft Excel-based system, the data of all patients who underwent cranioplasty in the neurosurgical OT were recorded. Data such as a patient's name, age, gender, registration number, date of surgery, the surgeon's name and operative diagnosis are kept in the system. Based on the inclusion criteria, patients who had undergone cranioplasty were selected and monitored post-operatively for surveillance of any surgical site infection development.

The inclusion criteria were as follows:

a. Traumatic brain injury patients were aged 12 years or older and had undergone cranioplasty.

The exclusion criteria were as follows:

a. Possibility of prior central nervous system infection, including subgaleal empyema, subdural empyema and cerebral abscess or possibility of prior infectious pathological disease, such as tuberculosis, toxoplasmosis or cryptococcal infection

b. Evidence of other systemic infection and/or antibiotic treatment

\section{c. History of infected cranioplasty}

All patients in the study population had their surgical site wound examined (direct observation of wound), ideally during their post-operative stay and through post-discharge surveillance (i.e., follow-up visits at the clinic two months, six months and one year after the operation). Medical and nursing records, information from clinical personnel and positive microbiology cultures were used as sources for potential identification of graft infection. Date, signs and symptoms of graft infection onset would be recorded on a surveillance form. Data would be collected using a cranioplasty graft infection 
form. The follow up was at least two months post-operation or post-discharge or transfer. It was assumed that patients suffering from a wound infection who were transferred to another hospital would be referred back to our unit. The respective medical officer in charge in the transferred hospital was informed about signs and symptoms of surgical site infection and was directed to contact us if there were signs and symptoms of graft infection. Patients included in the study who had been discharged were checked again in the neurosurgical clinic two months post-operation for post-operative graft infection. They were also taught about the signs and symptoms of infection and how to self-inspect the cranioplasty wound. Should they detect any signs or symptoms, they were instructed to come back to the hospital immediately.

The data collected included gender, Glasgow Coma Scale(GCS) score, craniectomy date, cranioplasty date, type of graft used, primary pathology, number of days elapsed between cranioplasty and craniectomy, number of surgical procedures pertaining to craniectomy site pre-cranioplasty and the name of the surgeon that performed the cranioplasty. The surgical site infections (graft infections) are classified according to the guidelines of the Centres of Disease Control. The dominator was the procedure, not the patients; in other words, cases of bilateral cranioplasty were recorded as two procedures. All patients who were included in this study gave their informed consent prior to the procedure. The study was awarded a National Medical Research Register number:NMRR: 17782, IRC-IIR/2014/08/048 from the Ministry of Health, Malaysia.

\section{Definition of surgical site infection (7-8)}

\section{Superficial incisional infection}

This is defined as a surgical site infection that occurs within 30 days of surgery, involves only the skin or subcutaneous tissue of the incision and meets at least one of the following criteria:

1. The superficial incision causes purulent drainage.

2. The superficial incision yields organisms from the culture of aseptically aspirated fluid or tissue or from a swab, and pus cells are present.

3. At least two of the following symptoms and signs are present: pain or tenderness, localised swelling, redness or heat.
The superficial incision is deliberately opened by a surgeon to manage the infection unless the incision is culture-negative, or the clinician diagnoses a superficial incisional infection. Stitch abscesses are defined as minimal inflammation and discharge confined to the points of suture penetration and localised infection around a stab wound. They are not classified as surgical site infections.

\section{Deep incisional infection}

This is defined as a surgical site infection involving the deep tissues (i.e., fascial and muscle layers) that occurs within 30 days of surgery if no implant is in place or within a year if an implant is in place. The infection appears to be related to the surgical procedure and meets at least one of the following criteria:

1. There is purulent drainage from the deep incision but not from the organ/space component of the surgical site.

2. The deep incision yields organisms from the culture of aseptically aspirated fluid or tissue or from a swab, and pus cells are present.

3. The deep incision spontaneously dehisces or is deliberately opened by a surgeon when the patient has at least one of the following symptoms or signs (unless the incision is culture-negative): fever ( $>38$ degree Celsius); localised pain or tenderness

4. An abscess or other evidence of infection involving the deep incision is found by direct examination during re-operation or by histopathological or radiological examination.

5. An attending clinician diagnoses a deep incisional surgical site infection.

Note: An infection involving both superficial and deep incision is classified as "deep incisional SSI" unless different organisms are present at each site.

\section{Organ/Space SSI}

This must meet the following criteria:

1. Infection occurs within 30 to 90 days after the operative procedure (where day $1=$ the procedure date) and involves any part of the body excluding the skin incision, fascia, or muscle layers, that is opened or manipulated during the operative procedure. 
2. The patient has at least one of the following: purulent drainage from a drain that is placed into the organ/space and/or organisms isolated from an aseptically-obtained culture of fluid or tissue in the organ/space and/ or an abscess or other evidence of infection involving the organ/space that is detected on direct examination, during invasive procedure or by histopathologic examination or imaging test and meets at least one criterion for a specific organ/space infection site.

\section{Results}

\section{Descriptive analysis}

Over a period of one year, a total of 189 cranioplasty cases were conducted in the Department of Neurosurgery at HKL. There were 105 cases of autologous bone cranioplasty and 84 of acrylic cranioplasty. A total of 172 cases fulfilled the criteria and hence were enrolled in the study. Seventeen cases were excluded because they involved re-cranioplasty procedures. Out of 172 cases, 105 (61.8\%) were autologous bone cranioplasty, and 67 (38.2\%) were acrylic cranioplasty. There was a high proportion of male patients undergoing cranioplasty: 118 (68.6\%) of the participants were male and 54 (31.4\%) were female. The primary pathology was due to trauma in the majority of patients (126 [73.2\%]) while only 46 cases $(26.8 \%)$ were non-trauma related.

Of the patients, 123 (71.5\%) had undergone a single cranial procedure prior to cranioplasty, and the balance (43 [28.5\%]) had undergone multiple cranial procedures. The majority of the patients (114 [66.3\%]) underwent cranioplasty 90 days after undergoing decompressive craniectomy; 58 (33.7\%) underwent cranioplasty less than 90 days after craniectomy. Most of the patients (137 [79.7\%]) presented with preoperative GCS higher than eight, and 35 (20.3\%) had GCS less than eight.

The infection rate in the period of study was noted to be $2.9 \%$. Three (4.5\%) of these infections involved acrylic, and two (1.9\%) involved autologous bone. Most of the implants were not affected (95.5\% of the autologous bone and $98.1 \%$ of the acrylic).

The prevalence of infection between the bone and acrylic groups was not significantly different $(P=0.379)$. The prevalence of infection between genders also showed no significant difference $(P=0.327[P<0.05])$. Testing was conducted to determine individual associations between the infection rate and other variables, including mechanism of injury, number of prior procedures, timing and GCS. The infection rates were not significantly different; the $P$-values were o.326, 0.140, 0.664 and 0.585 , respectively. All of these P-values are greater than 0.05; thus, the association between these variables and the infection rate was not significant.

\section{Discussion}

Graft infection in cranioplasty is a recurring problem. Despite advances in surgical technique and graft development, the rate of graft infection reported in the literature worldwide remains high (up to 33\%) (8-9). Various factors have been attributed to graft infection. Among them are the type of graft, pathology, number of prior procedures, time intervals, duration of surgery, and GCS pre-cranioplasty. Other factors, such as the method of bone preservation, also play a role in graft infection. The common methods of bone preservation are freezing the bone flap using cryopreservation or storing it within abdominal subcutaneous tissue. Various temperatures have been tried for optimal cryopreservation, ranging from -4 to $-85^{\circ} \mathrm{C}$. Temperatures that are too low are more costly and increase the risk of osteocyte loss and protein degeneration but, at very low temperatures, infection is less likely to happen. Higher temperatures may keep the osteocytes viable, but they also increase the risk of infection. Since no guidelines exist, various temperatures are used Malaysia's hospitals. Storage under abdominal subcutaneous tissue is cost effective, but patients have additional scars as a result of its use, and it may cause patients discomfort. It is also associated with bone flap resorption (10).

As noted above, this study's graft infection rate was $2.9 \%$. This figure is lower than reported figures worldwide (3-6). Among the reasons for this difference are this study's small sample size and its limited duration. The majority of the study's patients underwent autologous bone cranioplasty as opposed to acrylic implant cranioplasty. This could be a significant contributing factor to the low infection rate as use of acrylic implants is associated with a higher rate of infection. Furthermore, the use of autologous bone implants reduces the risk of immune and inflammatory reactions, which are commonly seen with acrylic implants. This reduces the potential development of post-operative edema and collections that can 
Brief Communication | Factors determining cranioplasty infection

Table 1: Association between demographics, graft, procedures, interval and infection

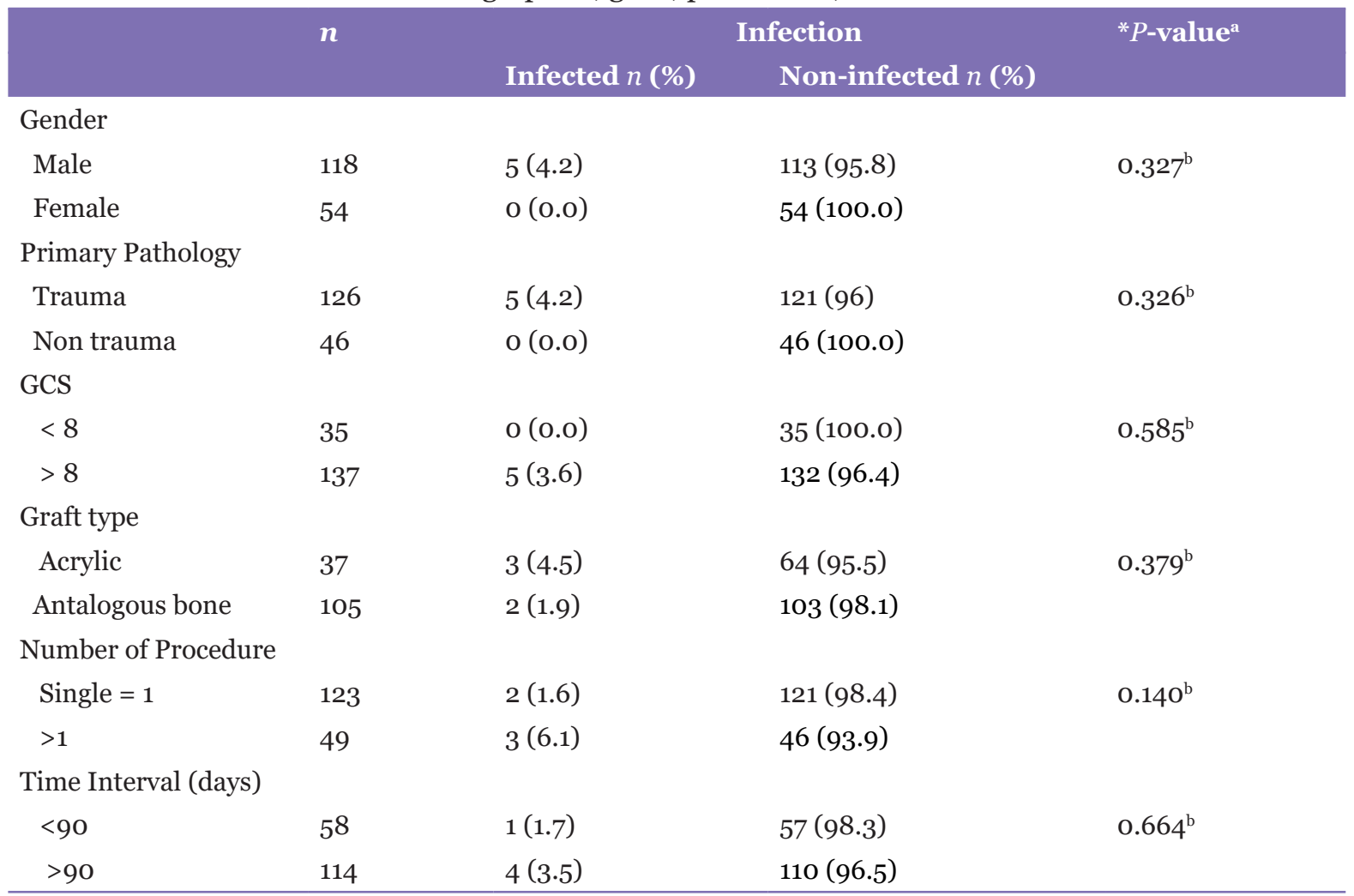

Notes: * $P$-value, were estimated either from Pearson Chi-Square or Fisher's Exact test. ${ }_{a}$ Chi-square test for independence ; ${ }_{b}$ Fisher's exact test

contribute to the development of graft infections in the future.

Patient selection also potentially played an important role in this study as the majority of patients were at low risk for the development of infection. Factors potentially reducing the risk of infection include a good preoperative GCS score, good health, nutrition and hygiene and a wellhealed craniectomy wound, all of which play an important role in wound healing. All cranioplasty procedures were performed in an elective controlled setting by a surgeon with more than three years of experience in neurosurgery and adequate knowledge and training with regards to neurosurgical procedures. This is an essential factor in reducing the potential development of intraoperative complications arising from poor surgical techniques. Furthermore, all cranioplasty procedures were performed in optimal operating room conditions with humidity levels of less than $50 \%$ to $60 \%$ and temperatures of 18 to $22^{\circ} \mathrm{C}$, with strict adherence to rules of sterility observed during the procedure.
The use of Poly(methyl methacrylate) (PMMA) for cranioplasty has been studied extensively. Methyl methacrylate (MMA) is a polymerised ester of acrylic acid the strength of which is equivalent to bone. Despite its advantages, MMA use carries a high risk of extrusion, decomposition and infection, and the residual monomer from cold polymerisation may be toxic. When MMA is ready for use, it is mixed with a monomer that causes an exothermic reaction. While this makes the substance a malleable paste, it also has the potential to cause burn injuries. The possible fragmentation of MMA leads to little protection in the long term and may even progress to infection and inflammatory reaction. Thus, a high incidence of infection and subcutaneous fluid collection has been reported (3). Regarding the relationship between graft types and infection rate, it was noted that rate of infection for autologous bone was $1.9 \%$ whereas acrylic had the higher rate of $4.5 \%$. However, the infection rates of the bone and acrylic groups were not significantly different $(P=0.379)$. This could be due to an inadequate 
sample size in the study that resulted in a suboptimal statistical analysis.

This is supported by the findings of Bobinski et al., who reported a lower infection rate in autologous bone grafts compared to that of artificial bone grafts (5). However, Matsuno et al. (6) found higher infection rates for both autologous bone (25.9\%) and PMMA (12.7\%) than for custom-made titanium mesh (2.6\%). Similar contradictory findings have been reported in the literature over the years. When analysing infection risk associated with graft materials, many factors have been debated by various authors. It was noted that the risk of infection from bone grafts was related to methods of preservation of the grafts. Findings favoured the storage of bone grafts within abdominal subcutaneous cavities over storage using cryopreservation; this conclusion was based on findings of a lower infection rate in subcutaneous implant bone and of the optimum storage temperature required to prevent development of infection in stored bone grafts. Other arguments have centred on the duration of storage of bone: a prolonged storage time leads to demineralisation of bone and protein breakdown, resulting in the formation of a nidus that perpetuates infection or osteomyelitis in an implanted bone.

In this study, all five cases of infection were male. However, gender is not a significant factor in the prevalence of infection in the literature $(5,6)$ This study's findings may have been caused by the greater number of men in its sample population.

The primary pathology of all five infected cases was from trauma. Previous infection due to a penetrating, open head injury appears to be an important risk factor for subsequent infection. However, when analysing the data, the infection rates do not significantly differ based on primary pathology. This may be due to an imbalance in the sample population: the majority of the study's cases resulted from trauma.

Within the first week following decompression, Cerebrospinal Fluid (CS) circulation derangements commonly manifest as subdural hygromas. There is a higher rate of wound infection in the early post-operative period after decompressive craniectomy. A contaminated wound on a trauma patient, emergency surgery or a large scalp flap with a narrow, long pedicle (which tends to injure the superficial temporal artery and open up the frontal air sinus) are all factors leading to a higher rate of wound infection in the early postoperative period after this procedure. A sinking scalp flap, resorption of preserved bone, slower neurological recovery rates, persistent headache and hydrocephalus are the most frequent complications of decompressive craniectomy beyond one month (5). All of these late complications are reversed after cranioplasty. In this study, we attempted to identify the time interval between craniectomy and cranioplasty that was associated with the highest infection rate. The sample population was divided into two groups: cranioplasties performed less than 90 days after the procedure (Group 1) and those performed more than 90 days after the procedure (Group 2). Fisher's exact test showed no statistically significant difference between the subgroups. There was only one infection in Group 1 and four infections in Group 2. The results of our statistical analysis may have been affected by the limited number of cases, but the data suggest that late cranioplasty carries an increased risk of infection. Both our study and that of Matsuno et al. show that undergoing multiple procedures before cranioplasty increases the risk of infection (6). Multiple neurosurgical revisions may be related to disrupted wound healing. Four phases of wound healing occur in an orderly and overlapping fashion: hemostasis, inflammation, proliferation (or granulation) and remodelling (or maturation). Inadequate time intervals between craniectomy and cranioplasty and multiple prior procedures clearly destroy established tissue and angiogenesis, thus increasing the risk of infection. However, there is no significant association between time interval and infection.

When we studied the effect of the number of procedures undergone by our patients prior to cranioplasty, we found no significant relationship between the number of procedures and the likelihood of infection. However, multiple procedures disrupt the wound-healing process due to repeated tissue insults. They also cause repeated exposure to bacterial translocation during the surgical procedures. Reopening the same incision site within a short period of time will result in a weakened scar and lower the barrier to bacterial infection. Multiple surgeries are also associated with a higher risk of subgaleal collection, which leads to a higher risk of infection.

The relationship between a patient's neurologic condition at cranioplasty and infection has not been previously examined, with the exception of one study that failed to show a significant statistical association between neurologic status and graft infection (4). In this 
study, GCS pre-cranioplasty was observed; all five infected cases had a GCS rating higher than $8 / 15$. However, the inadequate sample size may have led to a suboptimal statistical analysis.

A patient whose neurologic condition was poor would likely have undergone multiple procedures before cranioplasty, making them susceptible to infection. Such patients also commonly presented with poor nutritional status, which may lead to poor wound healing and infection. In patients with poor neurologic status, prolonged bedrest is common, and this is also associated with poor wound healing due to local compression of surgical wounds and subgaleal collection. This may also lead to surgical site infection. This group of patients has also experiences prolonged hospital stays, which means they have been vulnerable to hospitalacquired infection and to prolonged usage of antibiotics (leading to antibiotic resistance that will make treatment of infections more challenging).

\section{Conclusions}

Complications following cranioplasty remain an unresolved issue despite advances in surgical techniques and graft material development. Among the reported complications, graft infection following cranioplasty remains a significant cause of morbidity. Various risk factors have been associated with the development of graft infection; among them are graft type, timing of cranioplasty and factors related to surgery. At HKL, the incidence of graft infection after cranioplasty was found to be $2.9 \%$. Among the factors analysed in this study, none had any significant association with infection. Identification of risk factors for graft infection is essential in order to improve patient care and reduce morbidity, mortality and the economic burden of health care. Post-operative review and surveillance is important to identifying risk factors for graft infection. Although many factors have been debated and attributed to infection following cranioplasty, such as graft material and timing of cranioplasty, the patient's overall health and nutritional and functional status also plays a significant contributory role in development of complications and morbidity following cranioplasty. This factor should be taken into foremost consideration when selecting patients for cranioplasty. Good patient selection, meticulous surgical techniques and strict adherence to sterility procedures are crucial in attempting to reduce the infection rate among cranioplasty patients.

\section{Correspondence}

Dr. Siti Azleen Mohamad

MD (USM), MSurg (Neurosurgery) USM

Institut Kajisaraf Tunku Abdul Rahman (IKTAR)

Hospital Kuala Lumpur

50586 Jalan Pahang

Wilayah Persekutuan Kuala Lumpur, Malaysia

Tel: +603 26155190

Fax: +603 26982598

E-mail: saffarineuro@yahoo.com

\section{References}

1. Shah Am, Jung H, Skirboll S. Materials used in cranioplasty: a history and analysis. Neurosurg Focus. 2014; 36(4):E19. http://dx.doi.org/10. 3171/2014.2.FOCUS13561

2. Carson LV, Goodrich JT, Prestigiacomo CJ. History of craniotomy, cranioplasty, and perioperative care. Neurosurg Focus. 2014;36(4): Introduction. http://dx.doi.org/10. 3171/2014.2.FOCUS1470.

3. Schildhauer TA, Bauer TW, Josten C, Muhr G. Open reduction and augmentation of internal fixation with an injectable skeletal cement for the treatment of complex calcaneal fractures. $J$. Orthop. Trauma. 2000;14(5):309-317. http:// dx.doi.org/10.1097/00005131-20000600000001

4. Ryu JI, Cheong JH, Kim JH, Kim CH, Kim JM. Delayed infection following cranioplasty: review of 4 cases. J Kor Neurotraumatol Soc. 2005;1(1): 110-113.

5. Bobinski L, Koskinen LO, Lindvall P. Complications following cranioplasty using autologous bone or polymethylmethacrylate-retrospective experience from a single center. Clin Neurol Neurosurg. 2013; 115(9):1788-1791. http://dx.doi.org/10.1016/j.clineuro.2013.04.013

6. Matsuno A, Tanaka H, Iwamuro H, Takanashi $\mathrm{S}$, Miyawaki S, et al. Analyses of the factors influencing bone grafts infection after delayed cranioplasty. Acta Neurochir. 2006;148(5):535540. http://dx.doi.org/10.1007/s00701-006-07406 
7. Horan TC, Gaynes RP, Martone WJ, Jarvis WR, Emori TG. CDC definitions of nosocomial surgical site infections 1992: a modification of CDC definitions of surgical wound infections. Infect Control Hosp Epidemiol. 1992;13:606-6o8. http://dx.doi.org/10.1017/So195941700015241

8. Horan TC, Gaynes RP, Martone WJ, Jarvis WR, Emori TG. CDC definitions of nosocomial surgical site infections, 1992: a modification of CDC definitions of surgical wound infections. Am $J$ Infect Control. 1992; 20(5):271-274. http:// dx.doi.org/10.1016/So196-6553(05)80201-9

9. Odom GL, Woodhall B, Wrenn FR. (1952).The use of refrigerated autogenous bone flaps for cranioplasty. J neurosurg. 1952;9(6):606-610. http://dx.doi.org/10.3171/jns.1952.9.6.0606
10. Prolo DJ, Burres KP, McLaughlin WT, Christensen AH. Autogenous skull cranioplasty: Fresh and preserved (frozen), with consideration of the cellular respond. Neurosurgery. 1979; 4(1): 18-29. http://dx.doi.org/10.1227/oooo6123197901000-00005

11. Bell WH. Resorption characteristics of bone and bone substitutes. Oral Surg Oral Med Oral Pathol. 1964;17:650-657. http://dx.doi. org/10.1016/o030-4220(64)90372-X 